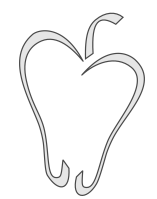

ISSN $1852-4249$

\title{
Physical pendulum experiment re-investigated with an accelerometer sensor
}

\author{
C. Dauphin,,$^{1,2 *}$ F. Bouquet ${ }^{3}$
}

\begin{abstract}
We have conducted a compound pendulum experiment using Arduino and an associated two-axis accelerometer sensor as measuring device. We have shown that the use of an accelerometer to measure both radial and orbital accelerations of the pendulum at different positions along its axis offers the possibility of performing a more complex analysis compared to the usual analysis of the pendulum experiment. In this way, we have shown that this classical experiment can lead to an interesting and low-cost experiment in mechanics.
\end{abstract}

\section{Introduction}

The physical pendulum experiment is the typical one to introduce the physics of oscillating systems. The usual aim of the analysis of this experiment is to determine the pendulum period and damping factor by using an angular position sensor $[1,2]$.

We have conducted the pendulum experiment by using a two axis accelerometer sensor. Such sensor has already been used by Fernandes et al. (2017) [3] but their study focused on the analysis of the time variation of the radial acceleration to investigate large-angle anharmonic oscillations.

Here, we have used the accelerometer to measure both radial and orbital accelerations of the pendulum at different positions along its axis, which offers the possibility of performing a more complex anal-

\footnotetext{
*E-mail: cyril.dauphin@villebon-charpak.fr

1 Institut Villebon-Georges Charpak, Université Paris-Sud (Bât. 490) - Rue Hector Berlioz - 91400 Orsay, France.

2 Département de Physique, University Paris-Sud, Université Paris-Saclay, 91405 Orsay cedex, France.

3 Laboratoire de Physique des Solides, CNRS, University Paris-Sud, Université Paris-Saclay, 91405 Orsay cedex, France.
}

ysis compared to the usual single measurement of the pendulum period.

Furthermore, we use a microcontroller and an associated two axis accelerometer sensor to acquire the data. Thus, we have used this simple and low cost experiment compared to the ready to use commercial one to introduce a richer theory and data analysis tools that can lead to an interesting experiment in mechanics.

We have described the theoretical analysis of this experiment and present an example of a possible experimental setup, the analysis of the measured radial and orbital acceleration in order to acquire the moment of inertia, the center of mass, the damping factor and the period of the pendulum.

\section{Example of experimental setup}

We use an Arduino [4] and a two-axis accelerometer sensor as measuring devices. The Arduino is an interesting choice for an experiment, as it is an easyto-use and low-cost microcontroller, with a large user community. Even if Arduino was not initially developed as a physicist tool, it can be used in various contexts of experimental physics activities (e.g., see references [5-10]). 
Papers in Physics, vol. 10, Art. 100008 (2018) / C. Dauphin et al.

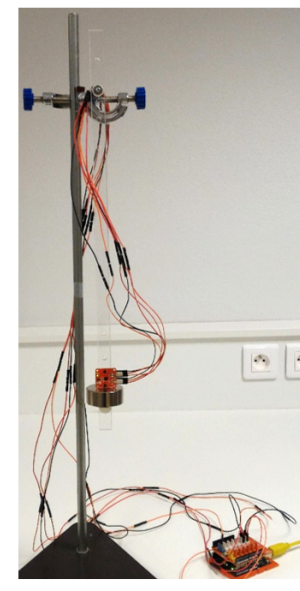

(a)

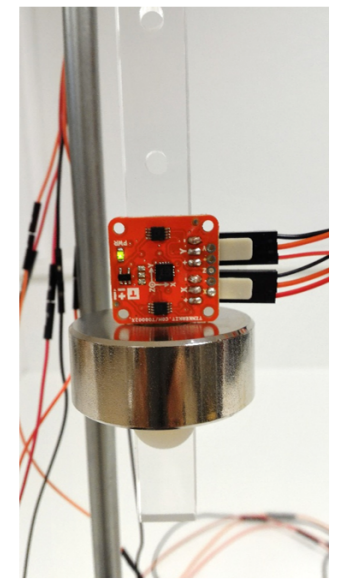

(b)

Figure 1: (a) An example of an experimental setup. (b) An accelerometer sensor attached to the centerline of the pendulum, with one of its axes parallel to the centerline.

The experimental setup that we used here is shown in Fig. 1(a). The accelerometer sensor is a microelectromechanical inertial sensor which is precisely calibrated by using the values $+g, 0 g$ and $-g$ for each axis with $g=9.8 \mathrm{~m} \mathrm{~s}^{-2}$.

The pendulum used in this experiment is composed of a bar on which masses can be attached to different positions. The accelerometer is attached to the bar and positioned in such a way that one of its measurement axes lies parallel to the bar (Fig. 1(b)). Special care should be taken so that the wires connecting the accelerometer to the board are flexible enough in order not to damp the pendulum.

Figure 2 shows an example of data acquired by the accelerometer. The main features of the graph are:

- the radial acceleration measurement decreases and goes to $g$ as $t$ approaches the infinity.

- when the radial acceleration reaches its maximum values, the orbital acceleration is essentially equal to zero (inset of Fig. 2).

- the radial acceleration is asymmetric about the straight line $a=g$ contrary to the orbital acceleration that is symmetric about the line $a=0$.

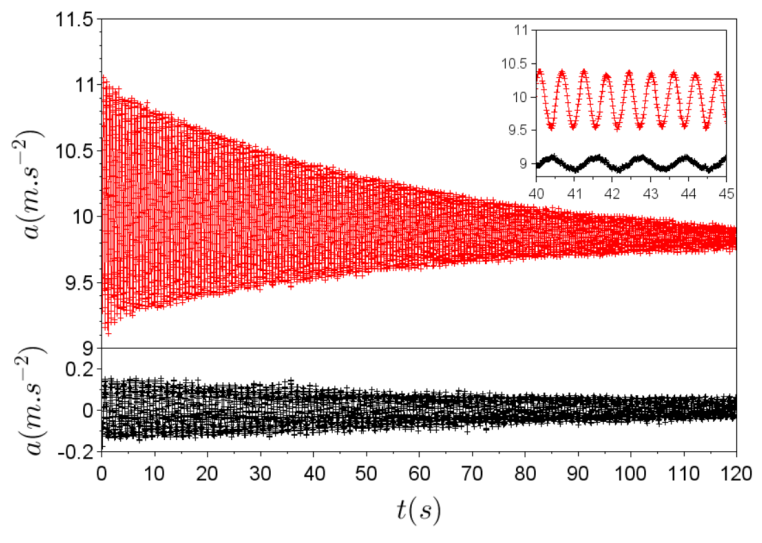

Figure 2: Radial (red) and orbital (black) accelerations measured by the accelerometer obtained with the experimental setup displayed in Fig. 1. Inset shows the temporal evolution of both accelerations between $40 \mathrm{~s}$ and $45 \mathrm{~s}$. In order to have both curves on the same plot, orbital acceleration is shifted by a constant offset of 9 in the inset.

- the period of the radial acceleration oscillations is twice that of the orbital acceleration oscillations (inset of Fig. 2).

In the next section, we will present the theory that explains these main features.

\section{Theory}

\section{i. Expression of the acceleration compo- nents measured by the accelerometer sensor}

Figure 3 shows a pendulum sketch with notations that will be used throughout this paper. $O$ is the pivot point, $G$ is the pendulum mass center and $A$ the accelerometer position. $L$ and $r$ stands for the distance between $O$ and $G$ and $O$ and $A$, respectively. We note $I_{\lambda}$ the moment of inertia of the pendulum about the $O z$ axis.

Applying the angular momentum theorem to the pendulum and considering viscous damping leads to:

$$
I_{\lambda} \ddot{\theta}=-M g L \sin \theta-\gamma \dot{\theta}
$$

where $\theta$ is the angle between the pendulum axis $O G$ and the vertical axis, $M$ is the mass of the system and $\gamma$ is the coefficient of friction. We note 


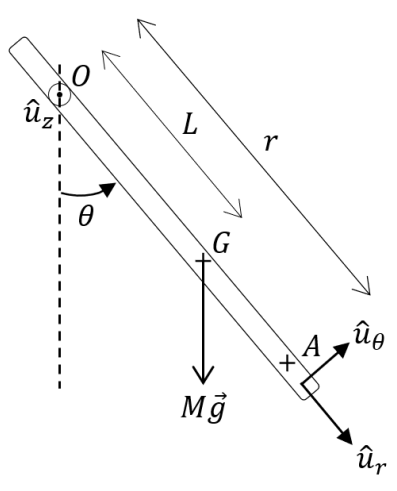

Figure 3: Sketch of the pendulum and notations used in the text. The pivot is at $O$, the center of mass at $G$ and the accelerometer sensor is at $A$.

$I_{\lambda}=\alpha M L^{2}$ where the numerical factor $\alpha$ depends on the type of pendulum ( $\alpha=1$ for a simple pendulum and $\alpha \neq 1$ for a physical pendulum). We introduce the damping factor $\kappa=\frac{\gamma}{2 I_{\lambda}}$ to obtain:

$$
\ddot{\theta}=-\frac{g}{\alpha L} \sin \theta-2 \kappa \dot{\theta}
$$

Forces acting on the proof mass $\left(m_{\text {sensor }}\right)$ inside the accelerometer are its weight and the inertial force due to its movement. Thus, the radial and orbital components of these forces in the non-inertial reference frame of the pendulum are given by:

$$
\begin{aligned}
& F_{r}=m_{\text {sensor }} r \dot{\theta}^{2}+m_{\text {sensor }} g \cos \theta \\
& F_{\theta}=-m_{\text {sensor }} r \ddot{\theta}-m_{\text {sensor }} g \sin \theta
\end{aligned}
$$

The acceleration components, as measured by the accelerometer at a distance $r$ from the pivot, are then given by:

$$
\begin{aligned}
& a_{r}=r \dot{\theta}^{2}+g \cos \theta \\
& a_{\theta}=-r \ddot{\theta}-g \sin \theta
\end{aligned}
$$

Including the expression of $\ddot{\theta}$ (Eq. (2)) into the expression of $a_{\theta}$ gives :

$$
a_{\theta}=\frac{g}{\alpha}\left(\frac{r}{L}-\alpha\right) \sin \theta+2 \kappa r \dot{\theta}
$$

We choose $\theta=\theta_{0}$ and $\dot{\theta}=0$ as initial conditions of the pendulum movement. We only consider here small damping $(\kappa<<\omega)$ and the small angle approximation $\left(\theta_{0}<<\frac{\pi}{2}\right)$, which leads from Eq. (2) to:

$$
\begin{aligned}
& \theta=\theta_{0} e^{-\kappa t} \cos (\omega t) \\
& \dot{\theta}=-\omega \theta_{0} e^{-\kappa t} \sin (\omega t) \\
& \ddot{\theta}=-\omega^{2} \theta_{0} e^{-\kappa t} \cos (\omega t)
\end{aligned}
$$

with the pendulum angular frequency $\omega=$ $\sqrt{\omega_{0}^{2}-\kappa^{2}}$ and $\omega_{0}=\sqrt{g / \alpha L}$. Thus, the acceleration components measured by the accelerometer for $\kappa<<\omega$ and $\theta_{0}<<\frac{\pi}{2}$ are given by:

$$
\begin{aligned}
a_{r} & =r \omega^{2} \theta_{0}^{2} e^{-2 \kappa t} \sin ^{2}(\omega t) \\
& +g \cos \left(\theta_{0} e^{-\kappa t} \cos (\omega t)\right) \\
a_{\theta} & =-2 \kappa r \omega \theta_{0} e^{-\kappa t} \sin (\omega t) \\
& +\frac{g}{\alpha}\left(\frac{r}{L}-\alpha\right) \sin \left(\theta_{0} e^{-\kappa t} \cos (\omega t)\right)
\end{aligned}
$$

With $\theta_{0}<<\frac{\pi}{2}$, we can further approximate these two expressions by:

$$
\begin{aligned}
a_{r} & =r \omega^{2} \theta_{0}^{2} e^{-2 \kappa t} \sin ^{2}(\omega t) \\
& +g\left(1-\theta_{0}^{2} e^{-2 \kappa t} \cos ^{2}(\omega t)\right) \\
a_{\theta} & =-2 \kappa r \omega \theta_{0} e^{-\kappa t} \sin (\omega t) \\
& +\frac{g}{\alpha}\left(\frac{r}{L}-\alpha\right) \theta_{0} e^{-\kappa t} \cos (\omega t)
\end{aligned}
$$

Some comments can be made about Eqs. (11) and (12). We first focus on the radial acceleration $a_{r}$.

- $\cos (\cos (x))$ and $\sin ^{2}(x)$ are both $\pi$-periodic functions. So $a_{r}$ is a $\frac{T}{2}$ periodic function with $T=\frac{2 \pi}{\omega}$ being the pendulum period. Indeed, the pendulum reaches its maximum velocity and so its maximum radial acceleration each time $\theta$ is equal to 0 .

- $g \cos \left(\theta_{0} e^{-\kappa t} \cos (\omega t)\right)$ varies between between $g \cos \left(\theta_{0}\right)$ and $g$ (blue curve in Fig. 4(b)) and goes to $g$ as $t$ approaches infinity. Physically, this function represents the projection of $\vec{g}$ onto the pendulum axis $O G$.

- $r \omega^{2} \theta_{0}^{2} e^{-2 \kappa t} \sin ^{2}(\omega t)$ varies between $r \omega^{2} \theta_{0}^{2}$ and 0 and the upper envelope of this function (red 
Papers in Physics, vol. 10, ART. 100008 (2018) / C. Dauphin et al.
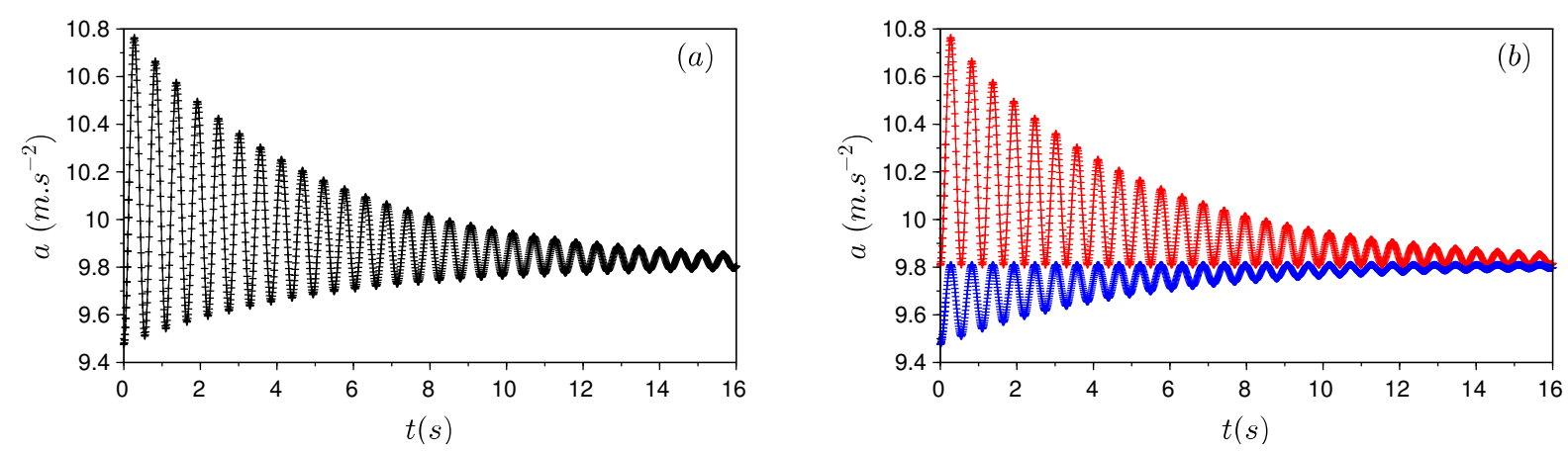

Figure 4: (a) Calculated radial acceleration versus time. (b) In red, graph of the $r \omega^{2} \theta_{0}^{2} e^{-2 \kappa t} \sin ^{2}(\omega t)+g$ contribution; in blue, graph of the $g \cos \left(\theta_{0} e^{-\kappa t} \cos (\omega t)\right)$ contribution. $L=30 \mathrm{~cm}, \theta_{0}=15^{\circ}, r=45 \mathrm{~cm}, \kappa=$ $0.1 \mathrm{~s}^{-1}$ and $\alpha=1$ for both panels.
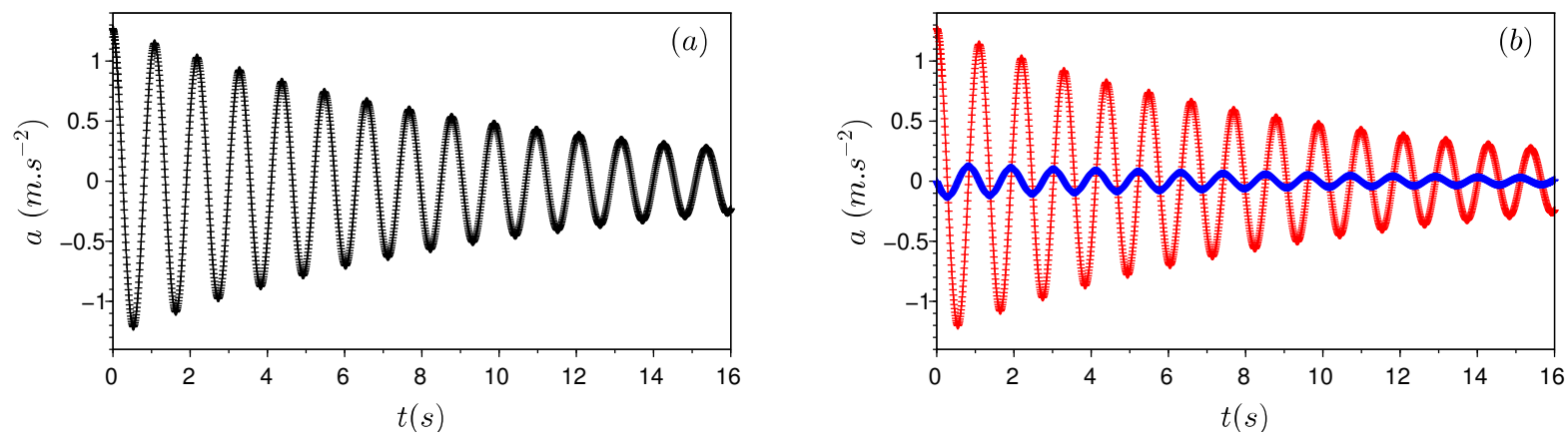

Figure 5: (a) Calculated orbital acceleration versus time. (b) In red, graph of the $\frac{g}{\alpha}\left(\frac{r}{L}-\alpha\right) \sin \left(\theta_{0} e^{-\kappa t} \cos (\omega t)\right)$ contribution; in blue, graph of the part $-2 \kappa r \omega \theta_{0} e^{-\kappa t} \sin (\omega t) . L=30 \mathrm{~cm}, \theta_{0}=15^{\circ}, r=45 \mathrm{~cm}, \kappa=0.1 \mathrm{~s}^{-1}$ and $\alpha=1$ for both panels.

curve in Fig. 4(b), note that it has been displaced by $g$ ) decreases exponentially as $e^{-2 \kappa t}$. Physically, this function represents the acceleration due to the radial centrifugal force felt by the sensor (as expected, the acceleration due to the radial centrifugal force is maximum when the pendulum is vertical). This point and the previous one explains the asymmetry of the radial acceleration about the straight line $a_{r}=g$, as observed in Fig. 2.

We can now study the orbital acceleration $a_{\theta}$ :

- $\sin (\cos (x))$ and $\sin (x)$ are both $2 \pi$-periodic functions. Therefore, $a_{\theta}$ is a $T$-periodic func- tion with $T=\frac{2 \pi}{\omega}$. Indeed, the angular acceleration $\ddot{\theta}$ reaches its highest and lowest values each time the pendulum passes through its highest points. This point explains that the period of the radial acceleration is twice that of the orbital one.

- $\frac{g}{\alpha}\left(\frac{r}{L}-\alpha\right) \sin \left(\theta_{0} e^{-\kappa t} \cos (\omega t)\right)$ goes to 0 as $t$ approaches infinity and the $\sin (\cos (x))$ function implies that the upper and lower envelope of this part of the $a_{\theta}$ function are symmetric about the straight line of equation $a=0$ (red curve in Fig. 5(b)).

- $-2 \kappa r \omega \theta_{0} e^{-\kappa t} \sin (\omega t)$ goes to zero as $t$ ap- 
PAPers in Physics, vol. 10, ART. 100008 (2018) / C. Dauphin et al.

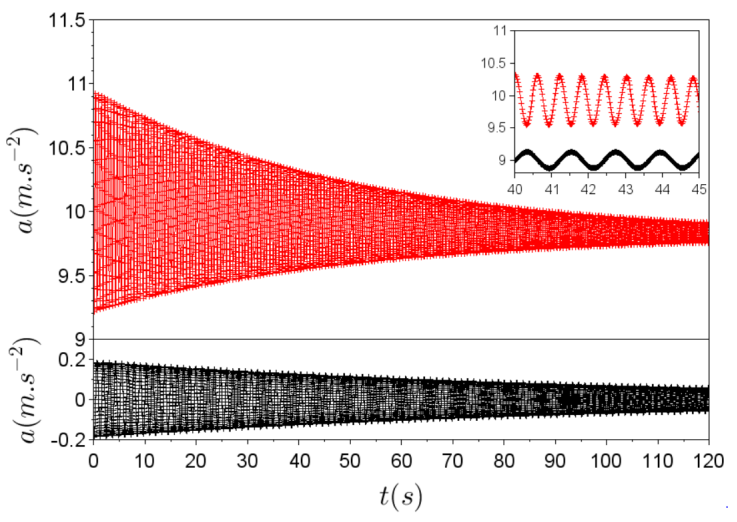

Figure 6: Radial (red) and orbital (black) accelerations obtained from Eqs. (11) and (12) with $\theta_{0}=20^{\circ}, L=$ $30 \mathrm{~cm}, r=34 \mathrm{~cm}, \alpha=1.2$ and $\kappa=0.01 \mathrm{~s}^{-1}$. Inset shows the temporal evolution of radial (red) and orbital (black) accelerations between $40 \mathrm{~s}$ and $45 \mathrm{~s}$. In order to have both curves on the same plot, orbital acceleration is shifted by a constant offset of 9 in the inset.

proaches infinity. The upper and lower envelopes of this part of the $a_{\theta}$ function are symmetric about the straight line $a_{\theta}=0$ (blue curve in Fig. 5(b)) and decrease exponentially as $e^{-\kappa t}$.

Figure 6 displays the radial (red) and orbital (black) accelerations obtained from Eqs. (11) and (12). We can see that the main features inferred from the data (Fig. 2) are well reproduced.

\section{ii. Experimentally accessible quantities}

The parameters describing the pendulum and its motion can be derived from the measurements of $a_{r}$ and $a_{\theta}$.

- $a_{r}(t=0)=a_{r, \min }=g \cos \theta_{0}$. Thus, the measured value of $a_{r}$ at $t=0$ leads to the value of $\theta_{0}$.

- A fit to the measured $a_{r}$ upper envelope with an exponential function allows us to determine the damping factor $\kappa$ of the pendulum from Eqs. (11) and (12). While, for small deflection angles $\theta$, see Eqs. (13) and (14), exponential fit of any envelope of measured $a_{r}$ or $a_{\theta}$ allows us to determine the damping factor.
- The sensor position $O A$ can be measured with great accuracy. Thus, the position of the pendulum center of mass and moment of inertia are the two quantities which are difficult to determine experimentally. Here, we use the fit of the temporal evolution of $a_{r}$ and $a_{\theta}$ to determine the product $\alpha L$.

\section{iii. Impact of $\alpha$ and $r$ on the measured ra- dial and orbital accelerations}

Figure 7 displays the evolution of the radial and orbital accelerations with time for different values of the moment of inertia (from $\alpha=1$ (Panel (a)) to $\alpha=2.5$ (Panel (d))). At the difference of the radial acceleration, we can see that the orbital acceleration depends strongly on $\alpha$. Indeed, $a_{\theta}$ expression at $t=0$ leads to $a_{\theta}(t=0)=\left(\frac{r}{\alpha L}-1\right) g \sin \theta_{0}$, which is an inverse function of $\alpha$. We can also note that $a_{\theta}(t=0)<0$ if $\alpha>\frac{r}{L}$ and $a_{\theta}(t=0)>0$ if $\alpha<\frac{r}{L}$. Thus, value of $a_{\theta}$ at $t=0$ gives information on the $\alpha$ value.

Figure 8 shows the evolution of the radial and orbital accelerations with time for different values of $r$. The distance $O A$ increases from Panel (a) to Panel (d). As expected, the amplitude of the radial acceleration increases with larger $O A$ values as the centrifugal force acting on the proof mass increases and the amplitude of the orbital acceleration decreases with larger $O A$ values as the rate of variation of $\dot{\theta}$ decreases with this distance.

In particular, acceleration components measured by the accelerometer attached to the position of the point $O$ are given by:

$$
\begin{aligned}
& a_{r}=g \cos \left(\theta_{0} e^{-\kappa t} \cos (\omega t)\right) \\
& a_{\theta}=-g \sin \left(\theta_{0} e^{-\kappa t} \cos (\omega t)\right)
\end{aligned}
$$

In this case, the only force acting on the mass inside the accelerometer is its weight and the expressions of the acceleration do not depend on $\alpha$. Accelerometer sensor is used in this case as an angular position sensor.

We also note that the acceleration components measured by the accelerometer attached to the position $r=L \alpha$ are given by: 

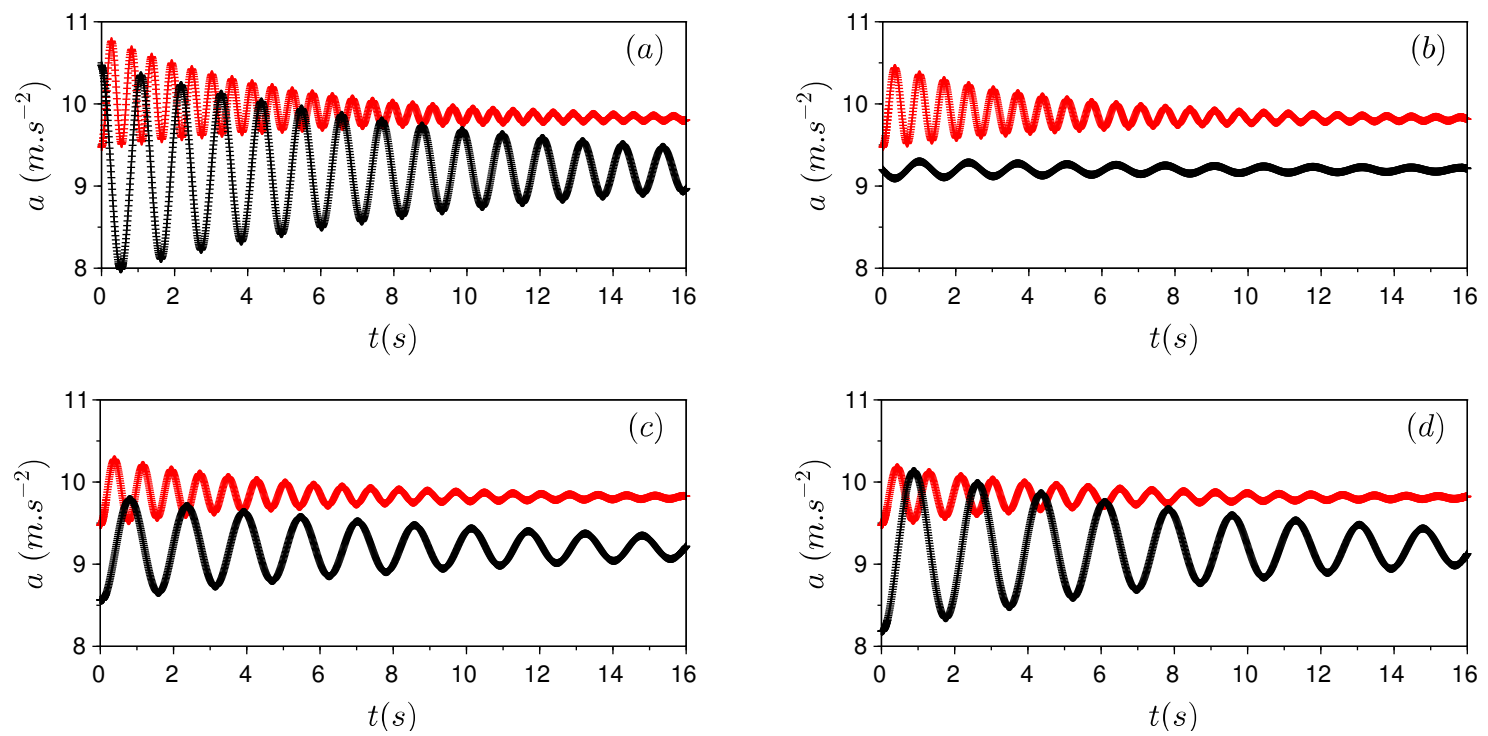

Figure 7: The calculated radial (red) and orbital (black) acceleration with time with $\alpha=1$ (a), $\alpha=1.5$ (b), $\alpha=2.0$ (c) and $\alpha=2.5(\mathrm{~d})$. In order to have both curves on the same plot, orbital acceleration is shifted by a constant offset of 9.2 . $L=30 \mathrm{~cm}, \theta_{0}=15^{\circ}, r=45 \mathrm{~cm}$ and $\kappa=0.1$ for all panels.
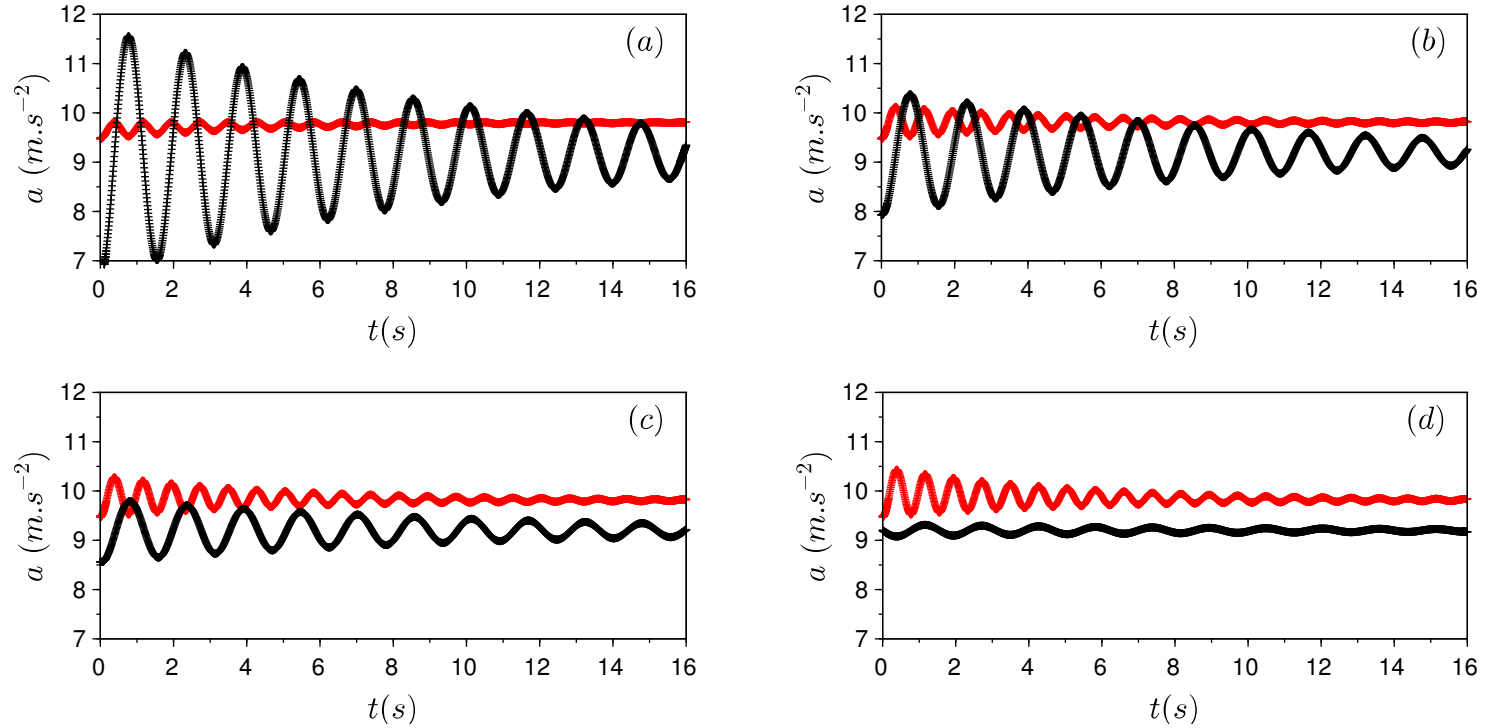

Figure 8: The calculated radial (red) and orbital (black) accelerations with time for different positions of the accelerometer relative to the center of mass $G$. In order to have both curves on the same plot, the orbital acceleration is shifted by a constant offset of 9.2. The accelerometer positions are (a) $r=0 \mathrm{~cm}$, (b) $r=30 \mathrm{~cm}$, (c) $r=45 \mathrm{~cm}$, (d) $r=60 \mathrm{~cm} . L=30 \mathrm{~cm}, \theta_{0}=15^{\circ}, \alpha=2$ and $\kappa=0.1$ for all panels. 

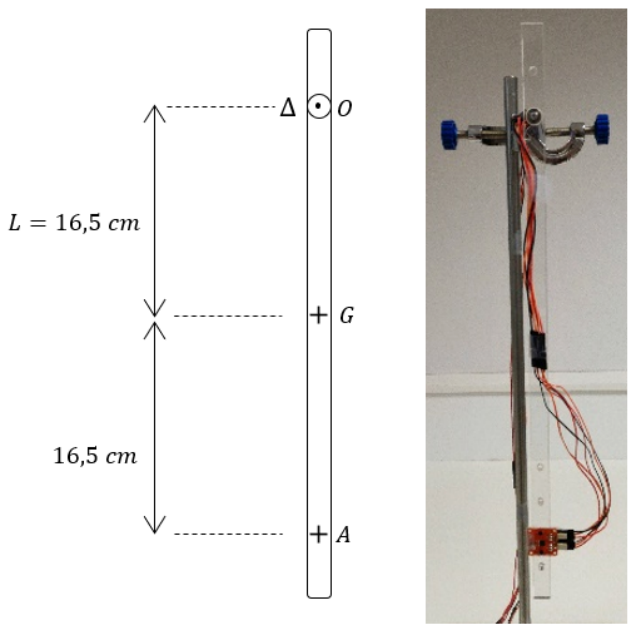

Figure 9: Experimental setup used to study a bar pendulum.

$$
\begin{aligned}
a_{r} & =\alpha \omega^{2} \theta_{0}^{2} e^{-2 \kappa t} \sin ^{2}(\omega t) \\
& +g \cos \left(\theta_{0} e^{-\kappa t} \cos (\omega t)\right) \\
a_{\theta} & =-2 \kappa \alpha L \omega \theta_{0} e^{-\kappa t} \sin (\omega t)
\end{aligned}
$$

In this case, component of $\ddot{\theta}$ due to the gravity force is counterbalanced by the orbital component of the force of gravity acting on the accelerometer sensor. Thus, $a_{\theta}$ is then directly proportional to the pendulum angular velocity.

After having shown that Eqs. (11) and (12) explain the features observed experimentally, we will now use them to retrieve the pendulum parameters $\kappa, \alpha$ and $L$ for different experimental setups.

\section{Example of data analysis}

\section{i. Example with a pendulum bar}

We first focus on the pendulum shown in Fig. 9 to derive its physical parameters. We use a bar of a $45 \mathrm{~cm}$ length and mass $45 \mathrm{~g}$ with the pivot at $16.5 \mathrm{~cm}$ from the center of mass and the accelerometer at $33 \mathrm{~cm}$.

Figure 10 shows the radial and orbital accelerations measured by the accelerometer after the pendulum has been displaced from the equilibrium position to an initial angle of $22^{\circ}$.

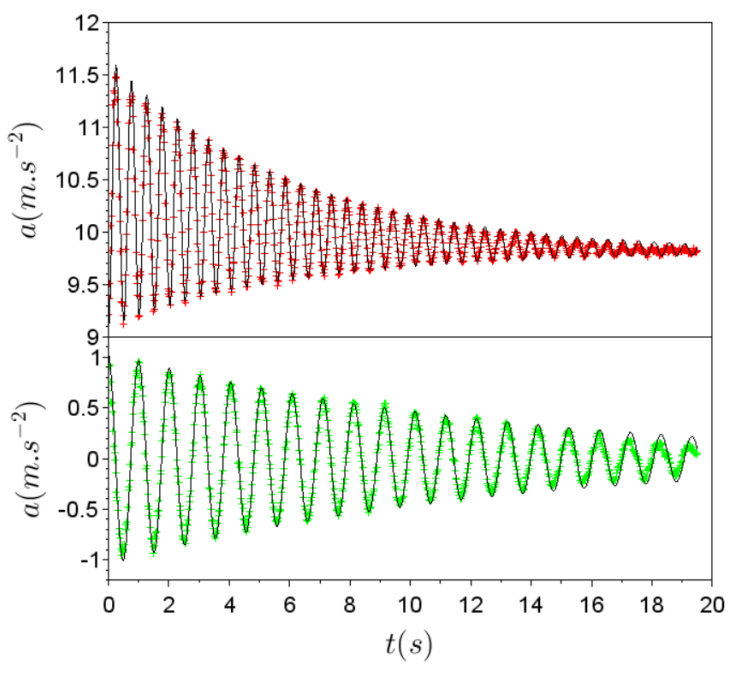

Figure 10: Radial (red) and orbital (green) accelerations measured by the accelerometer in the configuration shown in Fig. 9. Solid lines are the best fit of the data with $\kappa$ and $\alpha$ as free parameters. The best fit is given by $1 / \kappa=12.2 \mathrm{~s}$ and $\alpha=1.56$.

We have analyzed the data using Eqs. (11) and (12) with $\kappa$ and $\alpha$ as free parameters. The results of the fit are shown as black curves in Fig. 10. We have derived the best fit for $1 / \kappa=12.2 \mathrm{~s}$ and $\alpha=1.56$.

Assuming the pendulum to be a simple homogeneous slab, we calculate that the inertia moment of the bar about the rotation axis $\lambda$ is equal to $1.93 \times 10^{-3} \mathrm{~kg} \mathrm{~m}^{2}$, which leads to $\alpha=1.62$. The holes in the bar, which are used for attaching the masses, together with the mass of the accelerometer, explain the difference between this and with the $\alpha$ value obtained from the data fit. This experiment allows us to determine with a good accuracy the moment of inertia of the pendulum.

\section{ii. Retrieval of the mass center position}

The pendulum of the previous subsection is a symmetric bar, thus, its mass center position can be determined precisely. In the general case, the mass center position can be difficult to determine and we can fit the data by using Eqs. (11) and (12) with $\kappa$, $\alpha$ and $L$ as free parameters and infer the position of the center of mass.

As an example of such analysis, we use a pen- 
Papers in Physics, vol. 10, ART. 100008 (2018) / C. Dauphin et al.

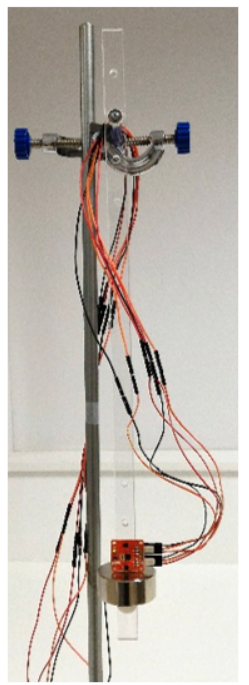

(a)

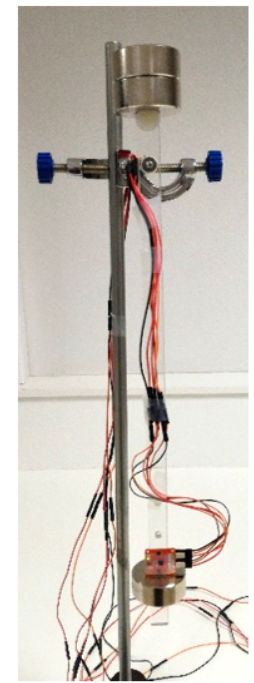

(b)
Figure 11: Experimental setup used to study compound pendula, with added masses.

dulum composed of a bar on which several masses can be attached to at different positions to acquired data that corresponds to pendulums of different values of $\alpha$ and $L$ (Fig. 11 (a) and (b)).

Figure 12 (a) and (b) displays the radial and orbital accelerations measured by the accelerometer after each pendulum shown in Fig. 11 has been displaced by an initial angle of $20.5^{\circ}$.

We fit the data by using Eqs. (11) and (12) with $\kappa, \alpha$ and $L$ as free parameters. Results of the fit are shown as the black curves in the insets of Fig. 12. Best fit are obtained with $1 / \kappa=111 \mathrm{~s}, \alpha=1.162$ and $L=0.293 \mathrm{~m}$ for the pendulum of Fig. 11(a) and $1 / \kappa=101 \mathrm{~s}, \alpha=6.55$ and $L=0.074 \mathrm{~m}$ for the pendulum of Fig. 11(b).

For the examples displayed in Fig. 12, the evolution of the orbital acceleration from Panels (a) to (b) shows an increase of $\alpha$, which is consistent with the fact that pendulum configuration goes from a configuration close to a simple pendulum $(\alpha \simeq 1$ Fig. 11(a)) to a compound pendulum $(\alpha>1$ Fig. $11(b))$.

Figure 12 shows that the angular acceleration (in green) is much more sensitive to the value of $\alpha$ than the radial acceleration (in red). Therefore, orbital acceleration is a good quantity to measure and to fit in order to determine the moment of inertia of a pendulum.

\section{iii. Impact of $r$ on the acquired data}

We have shown in section III that the orbital acceleration is very sensitive to the accelerometer sensor position with respect to the pendulum rotation axis. As this position is precisely known, we can perform several measurements with different positions of the accelerometer to improve the accuracy and/or the precision of the derived pendulum parameters. As an example of such analysis, we use the experimental setups shown in Fig. 13.

Figure 14 (a) and (b) display the radial and orbital accelerations measured by the accelerometer after each pendulum shown in Fig. 13 has been displaced by an initial angle of $20.5^{\circ}$.

Fits of the data using Eqs. (11) and (12) with $\kappa, \alpha$ and $L$ as free parameters are shown as black curves in Fig. 14. Best fit are obtained with $1 / \kappa=$ $111 \mathrm{~s}, \alpha=1.162$ and $L=29.3 \mathrm{~cm}$ for the pendulum of Fig. 13(a) and with $1 / \kappa=100 \mathrm{~s}, \alpha=1.162$ and $L=29.2 \mathrm{~cm}$ for the pendulum of Fig. 13(b).

The position of the accelerometer does not affect the calculated values of the moment of inertia of the pendulum and only slightly affects the center of mass position. The new configuration of the wires connecting the accelerometer in Fig. 13(b) changes slightly the $\kappa$ value. Thus, performing a second measurement with a different position of the accelerometer allow us to be more confident in the results retrieved from the first one.

We can also note that the orbital acceleration increases with lower $O A$ values, therefore the orbital acceleration fit precision is better when the accelerometer is in the position of Fig. 13(b), while the precision of the radial acceleration fit is better when the accelerometer is in the position of Fig. 13(a).

\section{Conclusions}

We have shown that the pendulum experiment analyzed with an accelerometer sensor leads to a theoretical study richer than the classical one. We have derived theoretical expressions for the radial and orbital acceleration data recorded by an accelerometer and separated the contributions from the pen- 
Papers in Physics, vol. 10, ART. 100008 (2018) / C. Dauphin et al.
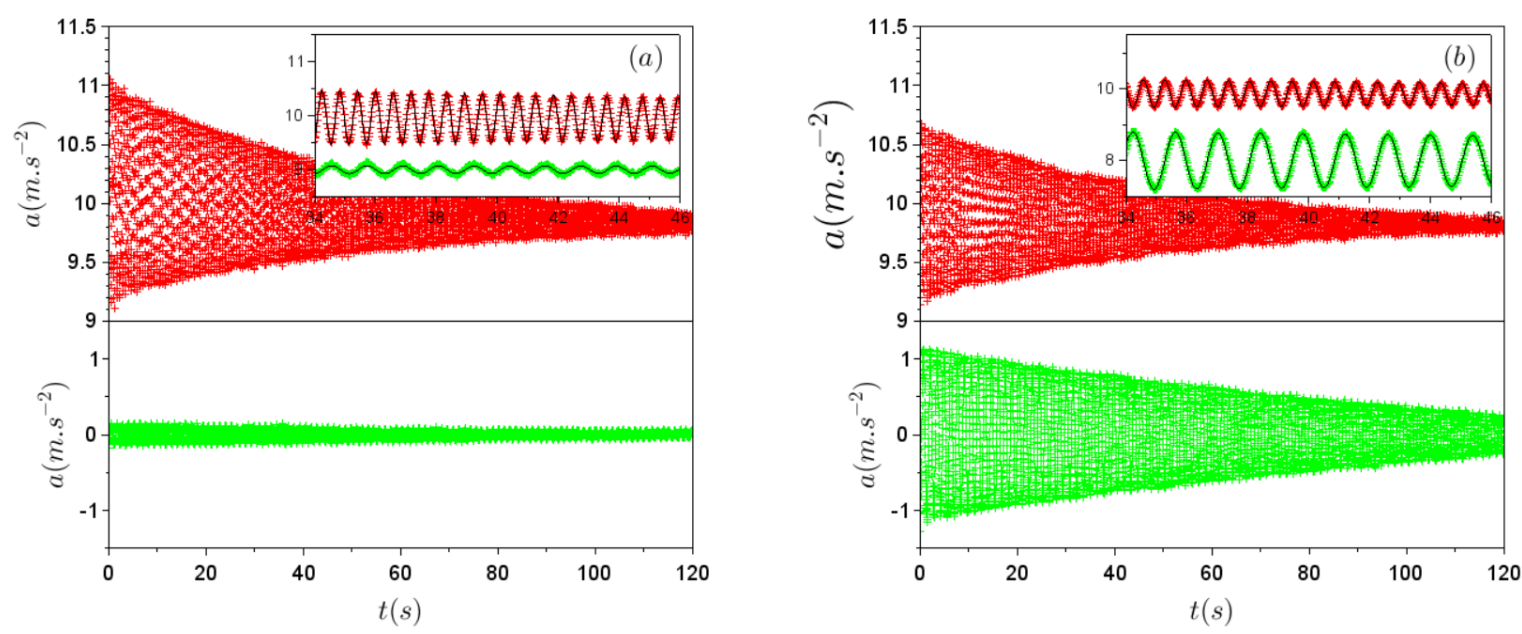

Figure 12: Radial (red) and orbital (green) accelerations measured by the accelerometer. (a) and (b) correspond to the setups displayed in Fig. 11 (a) and (b), respectively. Insets in (a) and (b) show the temporal evolution of the radial (red) and orbital (green) accelerations and the best fit (black) between $35 \mathrm{~s}$ and $45 \mathrm{~s}$. In order to have both curves on the same plot, orbital acceleration is shifted by a constant offset of 9 in the inset of (a) and by 8 in (b).

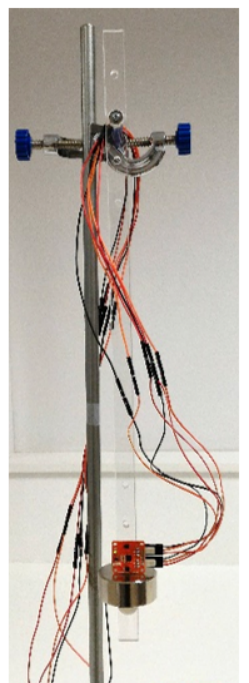

(a)

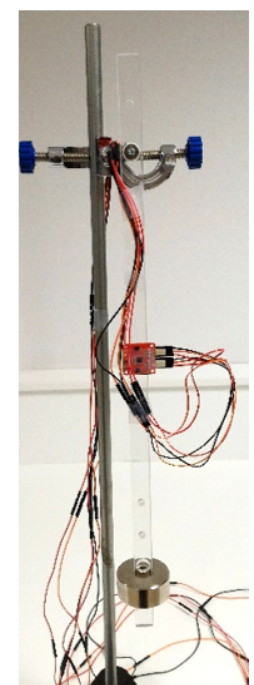

(b)
Figure 13: Experimental setup used to study the impact of the acceleration sensor position on the acquired data. dulum angular motion and the gravitational force on the proof mass.

We have also shown that the orbital acceleration is an interesting data to retrieve the moment of inertia of a pendulum.

The possibility to have different positions of the sensor allows us to perform several measurements with the same pendulum to improve the accuracy and/or the precision of the derived pendulum parameters.

In this paper, we have only focused on the classical pendulum but the device used here could also be applied to more complex systems such as chaotic pendulums.

Acknowledgements - This work benefited from a grant "Pédagogie innovante" from IDEX ParisSaclay. The Institut Villebon - Georges Charpak was awarded the Initiative of Excellence in Innovative Training in March 2012 (IDEFI IVICA: 11IDFI-002), and is supported by the Paris-Saclay Initiative of Excellence (IDEX Paris-Saclay: 11IDEX-0003). The authors would like to thank the referee for his/her helpful comments. 
Papers in Physics, vol. 10, ART. 100008 (2018) / C. Dauphin et al.
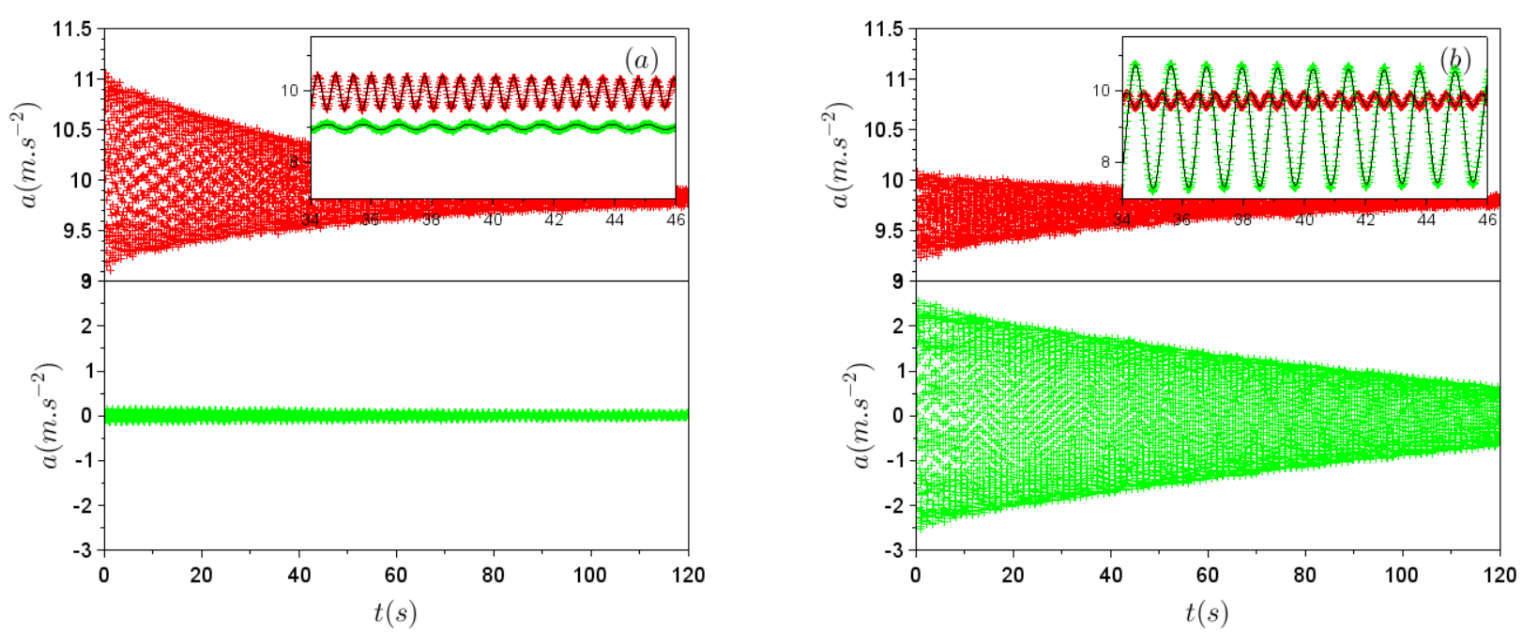

Figure 14: Radial (red) and orbital (green) accelerations measured by the accelerometer. (a) and (b) correspond to the setups displayed in Fig. 13(a) and (b), respectively. Insets in (a) and (b) show the temporal evolution of the radial (red), and orbital (green) accelerations and the best fit (black) between $35 \mathrm{~s}$ and $45 \mathrm{~s}$. In order to have both curves on the same plot, orbital acceleration is shifted by a constant offset of 9 in the insets of (a) and (b).

[1] http://www2.vernier.com/sample_labs/ PHYS-AM-18-physical_pendulum.pdf

[2] https://physics.fullerton.edu/files/ Labs/225/Physical_Pendulum_V4_1(1).pdf

[3] J C Fernandes, P J Sebastião, L N Gonçalves, A Ferraz, Study of large-angle anharmonic oscillations of a physical pendulum using an acceleration sensor, Eur. J. Phys. 38, 2017.

[4] https://www.arduino.cc

[5] Š Kubínová, J Šlégr, Physics demonstrations with the Arduino board, Phys. Educ. 50, 472 (2015).

[6] C Galeriu, S Edwards, G Esper, An Arduino investigation of simple harmonic motion, Phys. Teach. 52, 157 (2014).
[7] K Atkin, Construction of a simple low-cost teslameter and its use with Arduino and MakerPlot software, Phys. Educ. 51, 024001 (2016).

[8] C Petry et al., Project teaching beyond Physics: Integrating Arduino to the laboratory, In Technologies Applied to Electronics Teaching (TAEE), pp. 1-6, IEEE (2016).

[9] R Henaff et al., A study of kinetic friction: The Timoshenko oscillator, Am. J. Phys. 86, 174 (2018).

[10] F Bouquet, J Bobroff, M Fuchs-Gallezot, L Maurines, Project-based physics labs using low-cost open-source hardware, Am. J. Phys. 85, 216 (2017). 